WellBeing International

WBI Studies Repository

1985

\title{
The Imbalance Between Experiment and Theory in Biology: The Need for Theory-Directed Modeling
}

\author{
M. L. Fidelman \\ Virginia Commonwealth University \\ D. C. Mikulecky \\ Virginia Commonwealth University
}

Follow this and additional works at: https://www.wellbeingintlstudiesrepository.org/acwp_arte

Part of the Bioethics and Medical Ethics Commons, Laboratory and Basic Science Research Commons, and the Research Methods in Life Sciences Commons

\section{Recommended Citation}

Fidelman, M.L., \& Mikulecky, D.C. (1985). The imbalance between experiment and theory in biology: The need for theory-directed modeling. In M.W. Fox \& L.D. Mickley (Eds.), Advances in animal welfare science 1985/86 (pp. 203-220). Washington, DC: The Humane Society of the United States.

This material is brought to you for free and open access by WellBeing International. It has been accepted for inclusion by an authorized administrator of the WBI Studies Repository. For more information, please contact wbisr-info@wellbeingintl.org.

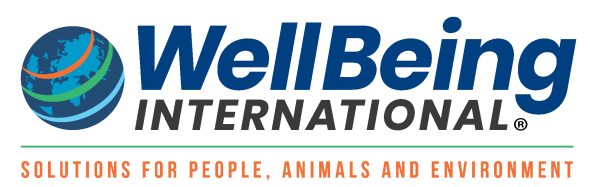




\title{
THE IMBALANCE BETWEEN EXPERIMENT AND THEORY IN BIOLOGY: THE NEED FOR THEORY-DIRECTED MODELING
}

\author{
M.L. Fidelman \\ D.C. Mikulecky
}

Department of Physiology and Biophysics

Medical College of Virginia

Virginia Commonwealth University

Richmond, VA 23298

\section{Introduction}

In biological and biomedical research, the vast majority of resources are focused on conducting experiments. Most of these experiments utilize animals. Only a tiny amount of resources is spent on theory and modeling. It is our contention and the basic theme of this paper that the imbalance between theory and experiment in biology produces very poor science. The implications of which are that many of the experiments conducted have little real scientific meaning or value and, therefore, go hand-in-hand with unnecessary animal use and suffering. Given the finite resources available for research, the redirection of significant resources from an almost entirely experimental approach into one with an emphasis on more theoretical and modeling activity will achieve a much better scientific result while considerably reducing the number of animals used in biological research.

Although the arguments developed here have a bearing on other issues as well, such as the optimum use of resources, future directions in the training of research biologists, etc., they have a very direct bearing on the rising awareness that the indiscriminant use of animals for any purely human purpose needs to be carefully reexamined. Without getting into the moral questions of whether animal research is justifiable (see Singer 1985), we will attempt, in this paper, to analyze the current philosophical structure of a field which consumes animals at a high rate. 
For these reasons, it is important for everyone to have some understanding of the philosophical underpinnings of modern biological research-its history, its present, and its future. This paper focuses on the relationship between experiment and theory in biology and the role that modeling plays in the quality and strength of this interaction. It concludes with a discussion of a relatively recent development, Network Thermodynamics. We believe that Network Thermodynamic modeling and computer simulation is the next step in providing a comprehensive theoretical framework for describing complex, hierarchical biological systems and will have a profound impact on the future of biology.

\section{The Scientific Method: Theory and Empiricism}

Empiricism is distinct from other epistemologies (theories of knowledge) in its almost total reliance on sense experience as the ultimate source of knowledge. As an approach to science, it represents the search for knowledge based solely on experiment and observation. Much attention is therefore focused on methods for collecting and processing data with the inherent belief that this collecting activity will lead to recognition of patterns and relationships between observed events and ultimately to our understanding of the laws of nature (Mahoney 1976). In contrast to this completely experimental approach, there is what we will call "theoretical deduction" which is based upon the ability of the human mind to deduce relationships between abstract, often seemingly unreal or imaginary entities, usually represented by mathematics. This is the completely theoretical approach and as a scientific methodology seeks to gain knowledge by reasoning from known principles to an unknown or by seeking a set of general principles which can then be applied to explain the specific. Its language is mathematics.

The strength of empiricism lies in its concrete attachment to the physical reality of the system being studied. Its weaknesses become more apparent and severe as the complexity of the system increases. In biological systems, for instance, where we often face multiple interactive, dynamic, nonlinear processes embedded within complex structures, it becomes virtually impossible to intuit the basic principles and theoretical framework by which the system operates from just "looking at the relationships" in the data. In fact, without a theoretical framework to start from, it is highly unlikely that the "critical" experiments necessary to eventually achieve understanding will even be performed. On the opposite side, theoretical deduction can provide the rigorous mathemati- 
cal framework for the development of basic principles leading to understanding. The weakness of disembodied theorizing, however, is the risk of total irrelevance, i.e., the mathematical formalism of the theory has little or nothing to do with the actual structures and processes in the system.

Given the match of the strengths and weaknesses of these two approaches, it should not be too surprising that the scientific method frequently offered as the best and most productive comes from the strong interaction of theory with experiment (Platt 1964; Murphy 1982). This brings us to the concept of a model. Jaynes (1976) tells us that the concepts of science are all concrete metaphors which serve to generate abstract concepts. He distinguishes between a theory and a model by pointing to the theory as a relationship of the model to the things a model is supposed to represent. For example, Bohr modeled the atom to resemble a miniature solar system. The theory would state all atoms are represented by this model. This may seem a subtle distinction and is certainly a subjective one, but nevertheless a useful one. Thus modeling may be thought of as the vehicle for the interaction between theory and experiment.

\section{Classification of Models}

It is important to realize that all biologists use models in formulating and interpreting experiments, whether or not they are consciously aware of it. Many models never become formulated beyond the state of pictures, diagrams or even merely being verbalized. Such inherently intuitive and nonanalytical descriptions we will simply call qualitative models. Models which involve specific mathematical descriptions will be divided into two main categories: empirical models and theory-directed models. By empirical, we mean a mathematical description of the input-output characteristics of a system that is not based on or derived from the actual structures and/or specific processes of that system. Empirical models are basically models of data and may be thought of as essentially either "black box" approaches or curve-fitting. Theory-directed models, on the other hand, are an attempt to mathematically describe the physical reality of a biological system at a sufficient level of complexity necessary to explain system behavior. While theory-directed models may have some empirical elements within them, they are ultimately directed towards developing a comprehensive theoretical framework for understanding both the holistic behavior of the system and the specific behavior and contribution of each of the component parts of the system. As opposed to models of data, theory-directed models are more models of systems and are sometimes capable of telling us something very important about reality without any specific experimental data. This is, obviously, the point of departure from pure empiricism. 


\section{Criteria for Judging Models}

If models are the vehicle for the interaction between theory and experiment, then the "quality" of the model becomes critical to the quality of the science produced. It becomes very important, therefore, to understand in a general sense the strengths and weaknesses of these three categories of models.

A model should be communicable since science is a community activity. Qualitative models, particularly the diagrammatic and pictorial ones, certainly have this quality. They are also simple to formulate and use and easy to alter. These qualities may have something to do with their being the most common type of model used in biological research. As the systems they describe become more complex, serious problems arise with their specificity, clarity, and rigor. At times, we are asked to help an experimentalist develop a theory-directed type of model to replace the qualitative model that they have been using. We typically find that the processes and mechanisms alluded to in their qualitative model do not have clear and specific descriptions. Quite often we also find that necessary intermediate steps have been completely left out or essentially ignored, although these steps may play an important role in regulating the overall system behavior. The biggest weakness with qualitative models is that they are untested (except perhaps in the mind of their developer). In other words, it is never demonstrated that a particular model can actually produce the type of behavior that it is supposed to explain, let alone make accurate predictions about the system's behavior under new conditions. An excellent example of this problem was shown by Eisenfeld and DeLisi (1984). They examined, on the basis of qualitative stability and instability, a number of qualitative models in the literature which described immune response regulation. Not too surprisingly, they found that a number of apparently plausible models were unstable and therefore were not viable models for the observed behavior. Thus qualitative models tend to produce a weak or nonexistent interaction between theory and experiment.

Empirical models are for the most part readily communicable and not too difficult to use or modify, although they are less so than qualitative models. Unlike qualitative models, they possess at least mathematical rigor in their definitions and descriptions and they are testable. They are good at codifying and organizing in a rigorous way the data sets for a particular system. For example, measurements are made under a given set of conditions and the data is used to calculate a number of parameters in the model. The experimental conditions are changed, new measurements are made, and again the particular parameters are calculated. How these parameters change under different conditions establishes a uniform way of discussing and analyzing the 
data between different labs investigating the same system. There are a number of weaknesses with this type of model. The first is that these parameters do not relate to the specific physical reality of the system. Some of the mathematical techniques used in this type of model (for example, compartmental analysis and equivalent circuit analysis) have a real physical interpretation in some very simple systems. In more complex systems, however, they no longer correlate with real physical parameters. Unfortunately, there is still the tendency to blur or ignore the original assumptions and incorrectly give these parameters a literal physical meaning. Second, since the parameters do not map one for one with the real system, there is usually a great deal of hidden information (the real variables of the system) buried in these parameters. This is why these models cannot be used to accurately predict system performance under new conditions but only to codify data between known conditions. Last, but not least, is the general inability of empirical models to provide fundamental insights into the behavior of complicated systems or to provide a fundamental theoretical framework because they do not correlate with the specific physical realities of the system and generally rely on "equations of convenience" which have little or no real scientific basis. The use of such equations may, in fact, make the model susceptible to violating basic scientific principles, such as conservation laws or the second law of thermodynamics. So we conclude that empirical models, while having some advantages over qualitative models, are also inadequate for producing the necessary interaction between theory and experiment.

Theory-directed models, like empirical models, also have the mathematical rigor for specificity and clarity in defining hypotheses and are also analytically testable as to their behavior. Because they are an attempt to model the physical reality of the system, they are not as "data dependent" as empirical models. They can, therefore, predict system behavior under new conditions as well as predict values of experimentally inaccessible variables or parameters. They are capable of exposing the contradictions in hypotheses as well as the contradictions and/or incompleteness of data sets. Insomuch as they utilize mathematical descriptions based on or derived from basic scientific principles, they can provide the fundamental theoretical structure for identifying the "essentials" of the system and provide general insights and knowledge into the functioning of the system. Their biggest weaknesses have been with their communicability, their difficulty of use, and their inadaptability. These are not so much inherent problems with theory-directed models as they are with the specific mathematical and analytical techniques that have been available to develop them. The most common approach has been using differential equations and solving them. As the models become more complex, this becomes a formidable task; solu- 
tions, if they can be obtained at all, become less and less transparent and often must be analyzed by computer. Finally, if closed-form solutions are not available, the problem is usually subjected to some numerical analysis technique. Such models tend to be difficult to eliminate even if they are shown to be incompatible with data, due to the labor involved in their creation, and they also can be difficult to modify for the same reason. Such techniques also require much time and effort to learn and are difficult to master. What is needed is a new technique or approach that can overcome these problems. Along with Chua (1969), we believe it must be a compromise between simplicity and reality. Along with Thom (1975), we will often ask it to be qualitatively faithful before it is made to be quantitatively so. His point, which needs to be taken seriously, is that often the shape of a predicted curve has more significance toward acceptance or rejection of a model than whether or not the model's prediction goes through one or more actual data points. Also, if possible, it must be testable by the most stringent tests available. We will have more to say about model verification later. Pictorial representations have always had value. If we can have a pictorial representation which also provides a paradigm for the analysis of holistic behavior while relating the holistic behavior to the various functional parts, we will have succeeded. We submit that the method we call Network Thermodynamics can provide a common language to bridge the gap between experimental and theoretical biology (Mikulecky 1983). We will present later some of the reasons why we believe this to be true. In the next sections we will discuss the current attitudes and practices found in biological research and their historical perspective.

\section{Current Attitudes and Practices}

Researchers in physics and chemistry, for example, are compelled to become familiar with both theoretical and experimental approaches. In a physics department at any major university, one finds a reasonable mix of theoreticians and experimentalists. The experimentalists have the quantitative background necessary to understand the theories and are expected to use them in designing and interpreting experiments. Unfortunately, in the life sciences, empiricism is the most widely accepted epistemology with only a very small subset of practicing biologists adopting the theoretical approach to some extent. This imbalance between empiricism and theory in biology has produced a number of serious and interrelated consequences.

Foremost, perhaps, is the growth of a literature full of data with weak interpretation, if any at all. Yates (1979), a former editor of the American Journal of Physiology, refers to it as, "... the routine, data-loaded 
papers without strong scientific context that constitute the bulk of those published in any biological field." Another discouraging result of this imbalance in biology is a communication gap of immense proportions between experimentalists and theoreticians characterized in part by a sense of isolation, frustration, and even distrust. The manifestations of this are many, but there is one type in particular which illustrates the closed-mindedness which has become almost fashionable as well as the misunderstanding of the issues involved. Often, good-intentioned meeting organizers bring together theoreticians and experimentalists at a meeting in the hope of breaking down the communication barriers and establishing some sort of common ground. An event which is all too common at such meetings is that after hearing some theoretical presentation, an experimentalist will introduce the talk he or she is about to present with sincere reassurances to their counterparts in the audience that the presentation is totally free of models and theory! The speaker then proceeds to describe a model which is both weakly defined and untestable, believing that the trap was really successfully escaped. This is a cheap shot in a way, because the type of model the experimentalist was avoiding was special, and the one inadvertently presented was so poorly formulated that it hardly qualified. We must at least grant that the distinction being made was valid.

It is important to realize that these attitudes are prevalent among those who define modern biology by deciding where the funding will go and by deciding who will be hired, promoted, and tenured as well as what will eventually become part of the literature and what else will go unpublished. That certain ideas, approaches, etc., are excluded is a natural and obvious consequence of this imbalance. This leads to the tendency for those who seek to pursue theoretical biology to be forced to obtain funds and justification for their existence by modeling the data produced by the "true" investigators - a more or less subservient service role. They are not asked to create theory, merely to model data and to help get it published by giving it some appearance of having been done for theoretical reasons. There is the suspicion that often biology is chosen as a field of study precisely because it is lacking the mathematical and theoretical rigor that seems so difficult to master in the other sciences. Murphy (1982), in a presentation at a conference on the Genetic Basis of the Epilepsies, comments on "... the deplorable divorce, between science and mathematics, that took place within the century or so since scholars were first allowed to believe that it is possible to be a serious scientist with an unsullied ignorance of mathematics. This I hold to be a capital mistake, partly because ignorance of mathematics narrows perspective, but mainly because it betrays what is the great strength of science, the mutually correcting influences of coherence and rigor on the one hand and empirical fact on the other." 
A more subtle result of this imbalance, but possibly equally as serious, is the creation of a kind of "work ethic" which places a high value on activity relating to data collection but little value on time spent "thinking" or developing theory. Many biological researchers are quite ready to learn some very difficult physical, chemical, engineering, and mathematical concepts in order to apply them to the design, testing, calibration, and use of instruments, but will think nothing of failing to do even the most rudimentary modeling of their hypotheses and collected data. This problem was stated very clearly by Rene Thom (1979) when he commented on the possibility of experimentalists in biology testing predictions from Catastrophe Theory models: "... I feel that we should not hurry for any 'experimental confirmation'; I think that a lot of theoretical thinking, of speculative modeling, has to be done before one might really start (our emphasis) to experiment to make a choice between models. Even so, it is doubtful that these experiments would interest very much present-day biologists, as they would be unable to understand their motivation. Quite likely, there is very little which can be done about the present situation: I agree with P. Antonelli (Transplanting a pure mathematician into theoretical biology, Proc. Conf. on Math., Stat., and the Environment. Ottawa 1974), when he states that theoretical biology should be done in Mathematical Departments; we have to let biologists busy themselves with their very concrete-but almost meaningless-experiments; in developmental biology, how could they hope to solve a problem they cannot even formulate?"

This work ethic is tightly coupled to the most crippling omission from the life of modern biologists, which is some guideline for judging quality. In the more "exact" sciences, mathematical theories provide a path from general truths to specific lines of investigation which are often absent or which are at best applied in the above-mentioned limited way in biology. The argument that it is justifiable to use animals in research because the research ultimately eliminates human (and sometimes animal) suffering depends very much on the quality of that research. There is certainly a legitimate case that can be made that the almost completely empiricist approach prevalent in modern biology cannot justify on the basis of quality the amount of money, resources, and animals that it consumes. The ethical aspects of using animals has been treated very adequately by others (Singer 1985).

\section{A Brief Historical Perspective}

The situation described to this point exists and it is the dominant means of doing biological and, especially, biomedical research today. How is it justified? How can it have happened? Gaylord Simpson once 
said that biology is the queen of all the sciences because it studies levels of complexity beyond those studied by physics and chemistry. It is the complexity of the living system that has been the reason so many biologists do not believe that the development of theory which was so integral in the practice of physics and chemistry could play the same role in biology. Theories for dealing with most systems simply failed to work in these complex systems. Both classical and nonequilibrium thermodynamics were chained to the "black box" approach to highly organized systems. It can be argued most strongly that for the understanding of most biological systems these approaches are both inadequate and inaccurate since they arose out of the conceptual framework of the analysis of so called "simple" systems: those which were homogeneous and relatively unstructured in their basic nature. To properly apply either classical or nonequilibrium thermodynamic reasoning to organized systems, it is necessary to apply them at the level of such homogeneous substructures and then use the proper method for combining the substructures into a functional whole. Often, what was to be called "theoretical biology" suffered from the problem of "throwing out the baby with the bath water." In order to get solutions, the mathematical and theoretical descriptions of biological systems were oversimplified to the point of no longer being a meaningful description of the real biological systems.

The old question of whether or not life can be described in terms of the principles of physics and chemistry has never been adequately answered. The illusion is that physics and chemistry are complete and all that biologists need to do is use their principles. Unfortunately, this is not the case. There is one strong philosophical idea underlying all of this: reductionism, the idea that by continuously subdividing a system to subsystems and then studying the subsystems in detail, the properties of the whole can be learned. Due to the dominant role of reductionism, there is a wealth of particle physics done at the expense of a macroscopic physics of highly organized systems. Chemistry through chemical physics and physical chemistry follows suit. Thus modern biology (as well as its predecessors) has one of two choices. Either we believe that reducing living systems to parts totally describable by physics and chemistry can explain everything or we resurrect vitalism. As in all paradoxes, there is a truth hidden. In fact, vitalism and the missing areas of modern physics and chemistry may be different ways of stating the same idea: living systems, among other, highly organized hierarchical systems, need a holistic approach based on a more holistic science, specifically one designed to deal with highly organized systems. The development of this science will be a breakthrough in physics and chemistry as well as biology. Meanwhile, theory and quantitative ideas are accepted as useful only insomuch as they help the reductionist drive 
to look at smaller and smaller pieces of the organism in the hope of understanding the whole more completely.

This process of reduction, so central to modern biology's mode of operation, is often attributed to Descartes and it is often called "Cartesian Reductionism." It is possible that if Descartes were alive today he would take strong issue with this identification. As well as being a philosopher, Descartes was also a biologist and mathematician. In his Rules for the Direction of the Mind, he exhorted that we need not investigate "what others have thought nor what we ourselves conjecture, but what we can intuit (our emphasis) clearly and evidently or deduce with certainty, for there is no other way to acquire knowledge." This, upon first examination seems to be a clear exhortation toward the empiricists' approach. However, the fact that he put so much stock in intuition must also be seen in the context of his ability to reason mathematically. Given this ability, he could easily see the need for the examination of the natural objects he sought to understand. But, where would he have been without the mathematical background? Later in the same work, he said, "Intuition is the undoubting conception of a pure and attentative mind, which arises from the light of reason alone, and which is more certain than deduction" (Kline 1980).

The so-called neo-romanticists, often identified with Theodore Roszak (Marx 1978), go far beyond this call for the use of intuition. Roszak (1979) speaks in terms of a holistic epistemology based on the use of human emotion as well as human reason to arrive at truth. While these views are generally vigorously rejected within the scientific community, we bring it up here because Roszak's holism is a reaction to the blind reductionism seen among so much of modern science and technology and as such makes a point which may be germane to the issue of animal rights. Roszak (1979) states, "... it became permissible for the scientist to admire the mechanical intricacy of nature, but not to love it as a living presence endowed with a soul and reflecting a higher order of reality. A machine can be studied zealously, but it cannot be loved." The suggestion is that the reductionist view allows its advocates to avoid facing the ethical questions by its mechanization and depersonalization of the objects of scientific investigation, especially those which are alive.

\section{An Example}

Before moving on to Network Thermodynamics, we would like to present an example to illustrate and summarize the various approaches that we have already presented. Suppose we present a television to a 
number of investigators (who have no previous knowledge of electronics) and ask them to explain how the TV works. The totally empiricist investigator would remove the back of the TV set and begin making measurements of currents, voltages, resistances, etc., ad infinitum. Given the complexity of a TV set (which is still far less than most biological systems), it seems extremely unlikely, if not impossible, that just collecting data will lead to an intuitive development of the network theory needed to understand the holistic behavior of the many subcircuits working together or the physics of the individual solid state components. The totally reductionist investigator might start by smashing the set with a sledge hammer and sorting out the pieces according to size, color, shape, where they end up after density gradient centrifugation, etc. They might then look at the carbon, silicon, and germanium crystals in an x-ray diffraction machine. However, a TV set, like many biological systems, is hierarchical in its organization. It can also be broken down along more functional lines, i.e., supplying power, amplifying signals, etc. The molecular or "cellular" detail may only be incidental to understanding its function from this perspective. While something of value may be learned about the components, it again seems virtually impossible to understand how the TV works by ignoring the "circuit schematic diagram." Finally, the investigator who totally uses theoretical deduction might watch the TV for a while and then go off and devise a very complicated, formal mathematical theory to explain how the TV works. While there may well be some very interesting general knowledge that can be gained from the theory, the chances that the theory will correlate well with what is actually going on inside the TV is again extremely remote.

We would hope that by this time it is apparent that the best approach is one which stresses the strong interaction of both theory and experiment. With this example we have the hindsight of knowing how the TV and its components work. We understand that network theory, electromagnetic theory, and solid-state physics are all equally necessary to fully comprehend how a modern TV works and perhaps we also have a sense of the experimental input that went into the development of these theories. The application of these theories to the design of new and very complicated devices is now done in the electronics industry with computers using circuit simulation programs. New ideas are first modeled this way before any bench work is done. When the model works, then the device is built and tested and it is only when both the model and the experimental device agree that the understanding is complete and it goes into production. With this process in mind, we proceed to Network Thermodynamics. 


\section{Network Thermodynamics Modeling and Computer Simulation}

About a decade ago, two independent proposals were made (Peusner 1983; Oster et al. 1971) to utilize the techniques of network theory as a tool to study complex interacting physicochemical systems such as those encountered in the description of biophysical processes. The innovative step was the realization that the formal structure of network theory was a good representation for other physical theories - thermodynamics in particular. In Network Thermodynamics, the topology of interactive systems, such as those in biology, is represented by means of interconnected network elements which dissipate, store, supply, or convert energy. In its "purest" sense it is an expansion of thermodynamic reasoning which uses concepts of topology, mainly graph theory, to include the structure or morphology of a system in its thermodynamic properties. In simple language, it allows us to "put together" into a functioning whole a lot of pieces we may have observed as parts of a complicated, organized system. This model of the functioning whole allows us to investigate the relationships between the whole and its parts. In a time when reductionist thinking is so prevalent, this is a significant conceptual step.

Network Thermodynamics is the product of the combination of thermodynamics with topological methods. It is important to realize that Network Thermodynamics is therefore completely independent from electronics and electrical network theory. In fact, electrical networks, which provide an excellent example of how the marriage of topology with the properties of single elements can be so very productive, can be considered as a special case of Network Thermodynamics in its broadest definition. Given the influence of reductionism in the basic sciences, it is perhaps not too surprising that the development of macroscopic approaches to highly organized, hierarchical systems became the domain of the engineering disciplines and was developed there first. In fact, it should be reassuring that this new approach readily and naturally incorporates what has been done in the past. It is not necessary to unlearn what we already know to utilize the added power of the network approach to organization. It is simply a matter of putting our existing thermodynamic notions into a broader framework which liberates us from most of the old constraints. Once we recognize, for example, that transport systems in membranes are capable of being phenomenologically described by Fick's law or a few, more complicated, relations such as the Michaelis-Menten scheme, we have a class of objects which are capable of being seen as "wired together" in a particular way in each distinct living system. A pattern develops and a network theory of life processes quickly emerges in the same way that electronic network theory evolved. 
In biology, we seek to identify and characterize functional units that are typically more elusive than those in a TV and often must rely more strongly on the obvious morphological or biochemical units as a starting point in our analysis. The same reasoning leads to the recognition of an alternative generalization of the closure and conservation properties we usually call Kirchhoff's laws when applied to electrical networks. By examining the way the flows traverse a compartmentalized tissue, a network of flows can be described which obeys a Kirchhoff flow law, and by similarly noticing that the driving forces for biological processes follow a closure property around closed loops in any system, a Kirchhoff's force law arises.

If we notice that the transporter in a biological membrane, the chemical reaction, and the electrical resistance are all alike in that they are governed by some flow-force relation and are responsible for dissipation in the system, we can begin to talk about a class of biological network elements which we might call "dissipators." When inertial effects are present, such as in the pulsatile movement of blood or its change of direction in a curved blood vessel, an inductive form of energy storage is present. In Network Thermodynamics, the point is that these objects divide a system into thermodynamic elements since they each handle energy in distinctly different ways and furthermore, there is a small set of categories of objects which exhaust the ways in which energy can be handled. Together with the topology, which includes the conservation and closure properties we can now call Kirchhoff's flow and force laws, the system is completely defined. What emerges is a clear, simple, rigorous way to analyze any system with complex structure, especially living systems. Thus, a living system can be visualized in terms of a schematic which consists of dissipators (resistors), storage elements (capacitors), and so on.

So far, the emphasis has been implicitly focused on the formulation of a description and analysis of the living system using Network Thermodynamics. This would only be a hope for the future if it were not for the progress already made, mainly in circuit theory, in providing for the analysis of such systems. For this purpose, a number of powerful computer simulation programs have been created and provide a means for such analysis. We currently use the circuit simulation program, SPICE2, to solve our biological networks. Eventually new programs will be created for biological simulation which will not require the translation of the system's "schematic diagram" into a pseudo-electrical language before they can be simulated. The use of these circuit simulation programs may lead to the notion that Network Thermodynamics is "merely analog computing." Analog models, according to Jaynes (1976), are not really scientific models in the sense that they do not necessarily act as a hypothesis for explaining or understanding the object of study. "Instead, 
the analog is at every point generated by the thing it is an analog of." If we accept this definition, it will be clear that the type of models we are discussing resemble analogs very closely, but indeed are mainly used to test hypotheses and do indeed aim at understanding or explaining the object of study. The scope and depth of Network Thermodynamics far exceeds analog computing.

Network Thermodynamic modeling techniques offer many unique advantages over other approaches currently in use. Network Thermodynamic simulation is easy enough to do to allow models to actually be investigated by their originators and discarded or modified with little loss in time or effort. Unlike techniques using only higher mathematics, the fact that Network Thermodynamics rests on schematic depictions of a system rather than an explicit formulation in terms of equations makes it far more accessible to the non-theoretician, while still retaining its complete mathematical rigor. It is intuitively easier to visualize physical processes using the drawings of network theory than the formalism of topology. The topology of networks adds a systematic method for specifying connections to the mathematics of physics and chemistry, which are basically poor in these relations. And most importantly, network theory provides algorithms for interconnecting and representing functional systems, whether linear or nonlinear, reciprocal or not, and allows an infinite number of possibilities to represent these systems on an organized basis. As such, networks serve to represent differential equations by discrete, computable algorithms. The availability of powerful (and cheap) circuit simulation computer programs, which can represent thousands of interconnected points, puts the power of network theory at the fingertips of practically anybody with an imaginative application. Specifically, some of the fields in which Network Thermodynamic modeling has begun to be used are the cellular pharmacokinetics of an anticancer drug, methotrexate (White 1979; White and Mikulecky 1982), filtration in the glomerulus of the kidney (Oken et al. 1981), whole body pharmacokinetics (Thakker et al. 1982), the effects of insulin on glucose transport and metabolism in adipocytes (May and Mikulecky 1982,1983), calcium uptake by the sarcoplasmic reticulum (Feher and Briggs 1982), volume and salt flow in the rat ileum (Fidelman and Mikulecky 1982) and kidney proximal tubule (Thomas and Mikulecky 1978), and hormone regulation of ion transport in cultured kidney epithelia (Fidelman and Mikulecky 1985).

\section{Model Verification}

One of the most brilliant scientists of our time was Aharon Katchalsky. He was one of the prime movers in the quest for a method for modeling 
the complex organization found in living systems. The quest for him as well as the quest for us ended with the discovery of Network Thermodynamics. Katchalsky was also a careful enough scientist to worry about the ability to create useless, unverifiable models. One of his fondest quotations was: "With four unknown parameters you can draw an elephant. With a fifth, you can also wag his tail."

This concern is, in part, a valid one. Fortunately, a large amount of work has now been done on the verification of models (Walter 1982: Carson et al. 1983; Mikulecky 1981, 1983). By model verification, we mean the evaluation of a model with respect to the uniqueness of its representation of the real system and to the one-to-one correlation of model parameters with the real system parameters at all the hierarchical levels in the model. So far, although this has already become a field in its own right, the scope of the results obtained is very limited. The kinds of models which are best evaluated by this body of work are linear compartmental models of small size. In some of our latest work, we try to show how Network Thermodynamics can bring in additional mathematical constraints on models to help characterize them more completely. Also, in those cases where more rigorous analytical methods fail, parameter estimation is possible by trial and error methods using simulation. Thus we can expect that Network Thermodynamics will continue to serve to help evaluate models both by analytical methods as well as simulation.

\section{Concluding Remarks}

In our discussion of Network Thermodynamics, we have focused more on the conceptual framework of this approach than on a practical "how to" guide. We refer the reader to Thakker et al. (1982), White (1979), White and Mikulecky (1982), May and Mikulecky (1982), and May and Mikulecky (1983) for some specific, and not too difficult examples of the application of these techniques. We would also welcome direct contact with anyone interested in learning more about using Network Thermodynamics. As a general rule, it is not our intent to become "expert modelers" for others, but to teach and assist those who wish to learn for themselves how to do theory-directed modeling using the Network Thermodynamic approach.

The predominance of empiricism and reductionism in modern biological and biomedical research has led to a situation where the finite resources available are mainly used to generate an overabundance of data with little, or no, real scientific meaning. This lack of a basic theoretical foundation in biology also produces little real scientific criteria for judging quality of research. In our opinion, the diversion of 
significant resources from experimental work into the development of theory and theory-directed modeling will achieve a much better scientific result from a considerably reduced number of experiments. It is our belief that in Network Thermodynamics we have the necessary framework to begin bridging the gap between theory and experiment in biology.

\section{Acknowledgements}

We extend our thanks and gratitude to Betty Lou LaJoy for her energy in being the catalyst behind the writing of this paper and for her insights into the importance of Network Thermodynamics in moving biological research in a new direction that will begin to be more compatible with animal rights. We would also like to thank Dr. Sheella Mierson for her asistance in reviewing this manuscript.

M.L. Fidelman is the recipient of a grant from the Whitaker Foundation. 


\section{References}

Carson, E.R., Cobelli, C. and Finkelstein, L. 1983. The Mathematical Modeling of Metabolic and Endocrine Systems: Model Formulation, Identification, and Validation. New York: Wiley.

Chua, L.O. 1969. Introduction to Non-linear Network Theory. New York: McGraw Hill.

Eisenfeld, J. and DeLisi, C. 1984. On conditions for qualitative instability of regulatory circuits with application to immunological control loops. Symposium on Mathematics and Computers in Biomedical Applications. NIH Publication No. 84-2705, p. 13.

Feher, J.J. and Briggs, F.N. 1982. The effect of calcium load on the calcium permeability of sarcoplasmic reticulum. J. Biol. Chem. 257:10191-9.

Fidelman, M.L. and Mikulecky, D.C. 1982. Network thermodynamic modeling of isotonic coupled flows in the rat ileum. Physiologist 25(4):334.

Fidelman, M.L. and Mikulecky, D.C. 1985. Network thermodynamic modeling of insulin and aldosterone stimulation of $\mathrm{Na}^{+}$transport in cultured renal epithelia. Biophys. J. 47:445a.

Jaynes, J. 1976. The Origin of Consciousness in the Breakdown of the Bicameral Mind. Boston: Houghton Mifflin.

Kline, M. 1980. Mathematics: The Loss of Certainty. New York: Oxford.

Mahoney, M.J. 1976. Scientist as Subject: The Psychological Imperative. Cambridge: Ballinger.

Marx, L. 1978. Reflections on the Neo-Romantic critique of science. Proc. Am. Acad. Arts and Sci. 107(2):61-74.

May, J. and Mikulecky, D.C. 1982. The simple model of adipocyte hexose transport: Kinetic features, effect of insulin, and network thermodynamic computer simulation. J. Biol. Chem. 257:11601-8.

May, J. and Mikulecky, D.C. 1983. Glucose utilization in rat adipocytes: The interaction of transport and metabolism as affected by insulin. J. Biol. Chem. 258:4771-7.

Mikulecky, D.C. 1981. Further uses of network simulation. In: Kuczera, J. Grygorczyk, C. and Przestalski, S. eds. Biophysics of Membrane Transport IV. Wroclaw, Poland: Agricultural Acad. Part II. p. 209-35.

Mikulecky, D.C. 1983. Network Thermodynamics: A candidate for a common language for theoretical and experimental biology. Am. J. Physiol. 245:R1-9.

Mikulecky, D.C. 1984. Network Thermodynamics: A simulation and modeling method based on the extension of thermodynamic thinking into the realm of highly organized systems. Math. Biosciences. 72:157-79.

Murphy, E.A. 1982. Muddling, meddling, and modeling. In: Anderson, V.E., Hauser, W.A., Penry, J.K., and Sing, C.F. eds. Genetic Basis of the Epilepsies. New York: Raven Press. p. $333-48$.

Oken, D.E., Thomas, S.R. and Mikulecky, D.C. 1981. A network thermodynamic model of glomerular dynamics: Application in the rat. Kidney Int. 19:359-73.

Oster, G.F., Perelson, A. and Katchalsky, A. 1971. Network Thermodynamics. Nature. 234:393-9.

Peusner, L. 1983. The principles of network thermodynamic theory and biophysical application. Lincoln, MA: Entropy. (Reprint of Ph.D. Thesis, Harvard Univ., 1970.)

Platt, J.R. 1964. Strong inference. Sci. 146:347-53.

Roszak, T. 1979. Science, knowledge, and gnosis. Proc. Am. Acad. Arts and Sci. 103(3):17-32.

Singer, P. 1985. Ten years of animal liberation. N.Y. Rev. Books. Jan. 17:46-57.

Thakker, K.M., Wood, J.H. and Mikulecky, D.C. 1982. Dynamic simulation of pharmacokinetic systems using the electrical circuit analysis program SPICE2. Comp. Prog. Biomed. 15:61-72. 
Thomas, S.R. and Mikulecky, D.C. 1978. A network thermodynamic model of salt and water flow across the kidney proximal tubule. Am. J. Physiol. 235:F638-48.

Thom, R. 1975. Structural Stability and Morphogenesis. Reading: Benjamin.

Thom, R. 1979. "Answer to Cristopher Zeeman's Reply." In: Dynamic Systems - Warwick, 1979. New York: Springer-Verlag. Lecture Notes in Mathematics \#468, p. 384-5.

Walter, E. 1982. Identifiability of State Space Models. New York: Springer-Verlag.

White, J.C. 1979. Reversal of methotrexate binding to dihydrofolate reductase by dihydrofolate: Studies with pure enzyme and computer modeling using network thermodynamics. J. Biol. Chem. 254:10889-95.

White, J.C. and Mikulecky, D.C. 1982. Application of network thermodynamics to the computer modeling of the pharmacology of anticancer agents: A network model for methotrexate action as a comprehensive example. Pharmacol. Ther. 15:251-91.

Yates, F.E. 1978. Good manners in good modeling: Mathematical models and computer simulation of physiological systems. Am. J. Physiol. 234:R159-60.

Yates, F.E. 1979. Comparative physiology: Compared to what? Am. J. Physiol. 237:R1-2. 